TITLE:

\title{
A negative ion beam application to artificial formation of neuron network in culture
}

\section{$\operatorname{AUTHOR}(\mathrm{S}):$}

Tsuji, Hiroshi; Sato, Hiroko; Baba, Takahiro; Gotoh, Yasuhito; Ishikawa, Junzo

\section{CITATION:}

Tsuji, Hiroshi ...[et al]. A negative ion beam application to artificial formation of neuron network in culture. REVIEW OF SCIENTIFIC INSTRUMENTS 2000, 71(2): 797-799

\section{ISSUE DATE:}

2000-02

URL:

http://hdl.handle.net/2433/39799

\section{RIGHT:}

Copyright 2000 American Institute of Physics. This article may be downloaded for personal use only. Any other use requires prior permission of the author and the American Institute of Physics. 


\title{
A negative ion beam application to artificial formation of neuron network in culture
}

\author{
Hiroshi Tsuji, ${ }^{a}$ Hiroko Sato, Takahiro Baba, Yasuhito Gotoh, and Junzo Ishikawa \\ Department of Electronic Science and Engineering, Kyoto University, Sakyo-ku, Kyoto 606-8501, Japan
}

(Presented on 6 September 1999)

\begin{abstract}
A negative ion beam modification of the biocompatibility of polystyrene surface was investigated for the artificial formation of neuron network in culture with respect to negative ion species. Negative ions of silver, copper or carbon were implanted in nontreated polystyrene (NTPS) dishes at conditions of $20 \mathrm{keV}$ and $3 \times 10^{15}$ ions $/ \mathrm{cm}^{2}$ through a mask with many slits of $60 \mu \mathrm{m}$ in width. For the surface wettability, the contact angle of ion-implanted NTPS was about $75^{\circ}$ for silver-negative ions, which was lower than $86^{\circ}$ of the original NTPS. For carbon implantation, on the contrary, the contact angles did not change from the original value. In culture experiment using neuron cells of PC-12h (rat adrenal pheochromocytoma), the cells cultured with serum medium in two days showed the cell attachment and growth in number only at the ion-implanted region on NTPS for all ion species. In another two days in culture with nonserum medium including a nerve growth factor, the outgrowth of neural protrusions was also observed only at the ion-implanted region for all ion species. There was a difference in number of attached cells for ion species. The silver-negative ion-implanted NTPS had a large effect for cell attachment compared with other two ion species. This reason is considered to be due to the lowest contract angles among them. (c) 2000 American Institute of Physics. [S0034-6748(00)62502-9]
\end{abstract}

\section{INTRODUCTION}

In the neuroscience field, a method to form an artificially designed neural network by cell culture in vitro is desired for investigation of information processing in biological neural systems. ${ }^{1-3}$ In the future surgical operation concerning nervous system, an interface between the silicon circuit and nerve network will be required in the development of advanced artificial arms and fingers. For the artificial nervenetwork formation, many researchers have been studying about extracellular matrices for guiding the growth direction of nerve fibers. In the direct patterning of inorganic substrates with a photolithography technique, the growth direction of nerve fibers could be guided along the pattern. ${ }^{2,3}$ In the photolithography, the photoresist was coated on the surface at first and was removed after deposition of collagen or metal oxide layer. The photolithography requires complex processes and may bring contamination or invasion to the surface for polymeric materials. Therefore, a simple and clean process is required.

Recently, the use of ion implantation into polymeric materials in the biomedical field has progressed and remarkable results have been obtained in improving biocompatibility ${ }^{4-7}$ and in conveying new biofunctionabilities such as selective attachment properties and cell orientation. ${ }^{7-9}$ And also, the ion implantation can modify the surface with a designed pattern by using a patterning mask. Therefore, the ionimplantation technique is considered to be very attractive method for surface modification in order to make an artificial neuron network on the polymer surface. In addition to all of

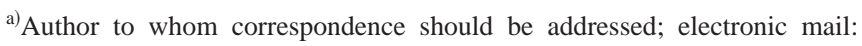
tsuji@kuee.kyoto-u.ac.jp
}

those, the ion implantation can dope desired elements of heavy metals with respect to studying the influence of heavy metals on neurons. The negative ion implantation has an advantage of "charge-up free" even in insulators and isolated electrodes. ${ }^{10,11}$ For this reason, we used negative ions as implantation species.

\section{EXPERIMENT}

\section{A. Negative-ion implantation into polystyrene}

Nontreated polystyrene (NTPS) dishes $(6 \mathrm{~cm}$ in diameter, No. 25060-60, Corning) were used as a base polymeric material and NTPS has no cell attachment properties on it. We implanted three kinds of negative ions: silver, copper and carbon, into this NTPS. Each of the negative ions was produced in and extracted from a cesium-sputter-type negative ion source [neutral and ionized alkaline metal bombardment-type negative ion source (NIABNIS) ${ }^{12,13}$ ] with a sputtering target made from a pure material $(99.99 \%$ silver, copper, or carbon) to be ionized. The negative ion beam was transported to an implantation chamber after being mass separated with an electromagnetic sector magnet at a bending angle of $30^{\circ}$. Figure 1 shows a schematic configuration of the negative ion-implantation chamber. NTPS dishes were placed on a baseplate of the collector cup and a mask was placed over the NTPS at a distance of $1 \mathrm{~mm}$. The mask was a nickel thin plate of $40 \mu \mathrm{m}$ in thickness and had 67 slits 60 $\mu \mathrm{m}$ wide and $4 \mathrm{~mm}$ long with a spacing of $60 \mu \mathrm{m}$ in a region of $4 \times 8 \mathrm{~mm}$. The ion beam passed through a limiter with a hole of $11.28 \mathrm{~mm}$ diameter and entered the NTPS surface through the mask in the collector cup. The implantation energies were 20, 15, and 10 (5) keV for silver-, copper- and 


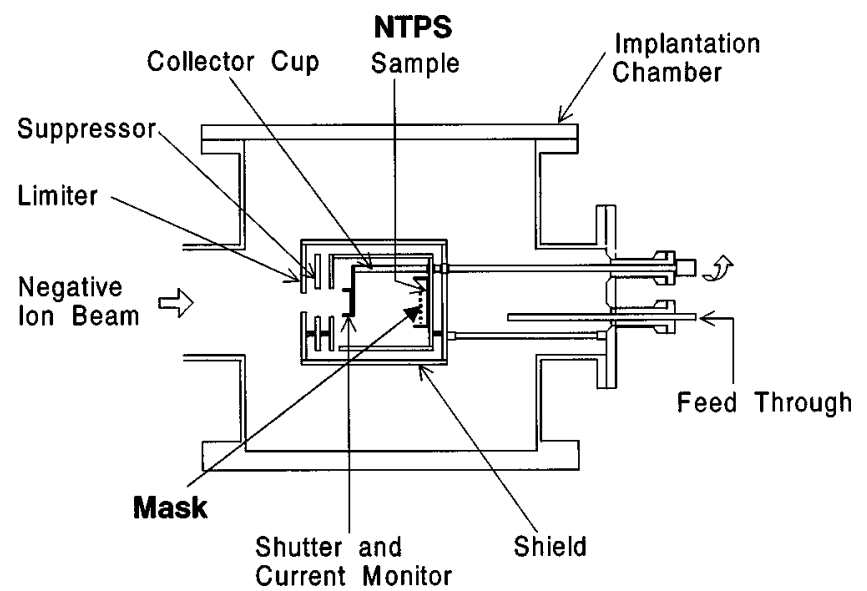

FIG. 1. Schematic configuration for patterned silver-negative ion implantation chamber.

carbon-negative ions, respectively, and the dose amount was a constant of $3.0 \times 10^{15}$ ions $/ \mathrm{cm}^{2}$ as for samples for a cellculture experiment. These energies were chosen in consideration for almost the same projected range. Current density was less than $200 \mathrm{nA} / \mathrm{cm}^{2}$. Residual and background gas pressures were about $1.0 \times 10^{-3} \mathrm{~Pa}$ and $1.4 \times 10^{-4} \mathrm{~Pa}$, respectively.

For contact angle measurement ${ }^{6-8}$ of the sample, negative ions were implanted into NTPS without the mask at conditions of $5-20 \mathrm{keV}, 1.0 \times 10^{14}-1.0 \times 10^{16} \mathrm{ions} / \mathrm{cm}^{2}$.

\section{B. In vitro cell culture experiment with PC-12h}

Nerve cells of PC-12h (rat adrenal pheochromocytoma) were used for in vitro studies. PC-12h cells respond reversibly to nerve growth factor (NGF) by differentiation into the neuronal phenotype (extension of neurites). The nerve cells expand neurites in the presence of NGF with nonserum medium in culture. Therefore, we can divide the culture period clearly into two stages: (1) growth of cells in number in a culture medium with serum but without NGF and (2) growth of neurite expansion in the presence of NGF with a nonserum medium. The former stage is related to the cell attachment and positioning, and the latter to network formation of neuron.

Pattern negative ion-implanted polystyrene dishes of NTPS ( $p$-Ag/NTPS, $p$-Cu/NTPS, $p$-C/NTPS) were sterilized for one day in $70 \%$ ethanol, and then rinsed three times by sterilized de-ionized water. PC-12h cells were seeded with a confluent amount on both sample dishes. In the first two days, the cells were cultured in a high-glucose culture medium (DMEM, Nissui), 5\% fatal bovine serum (FCS, BioWhittker), and 5\% heat-inactivated horse serum (FBS, ICN Biomedicals), with antibiotics in a humid, $5 \% \mathrm{CO}_{2}$ incubator at $37^{\circ} \mathrm{C}$. After the first two-day culture, the medium was replaced with the nonserum DMEM. The cells were cultured in the presence of $50 \mathrm{ng} / \mathrm{ml} \mathrm{NGF}$ with the nonserum DMEM in the incubator for another two days.

As a control sample in the cell culture, $40 \mu \mathrm{g} / \mathrm{ml}$ collagen-coated tissue-culture polystyrene (TCPS) dishes (6 $\mathrm{cm}$ in diameter, No. 25010-60, Corning) were used in the

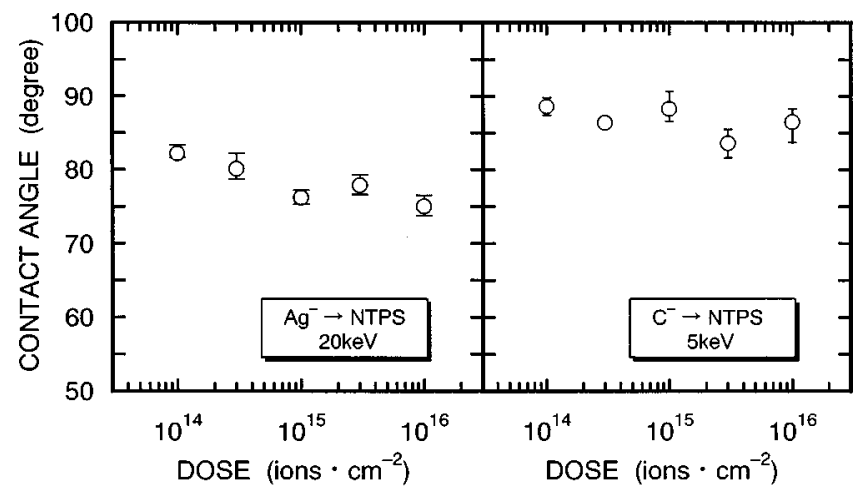

$\begin{array}{ll}\text { (a) } \mathrm{Ag}^{-}: 20 \mathrm{keV} & \text { (b) } \mathrm{C}^{-}: 5 \mathrm{keV}\end{array}$

FIG. 2. The change of contact angle as a function of the ion dose for (a) $\mathrm{Ag} / \mathrm{NTPS}$ at $20 \mathrm{keV}$ and (b) C/NTPS at $5 \mathrm{keV}$.

same culture experiment. Cell attachment and differentiation were observed by using a phase-contrast optical microscope after every $24 \mathrm{~h}$.

\section{RESULTS AND DISCUSSION}

\section{A. Contact angle}

Figure 2 shows the change of contact angle in negative ion-implanted NTPS as a function of the ion dose for (a) $\mathrm{Ag}^{-}$at $20 \mathrm{keV}$ and (b) $\mathrm{C}^{-}$at $5 \mathrm{keV}$. It is clear in silver-ion implantation that the contact angle was decreased down to $74^{\circ}$ from $86^{\circ}$ for the original surface with increasing ion dose. The similar dependence was also observed in the copper-negative ion implantation at $15 \mathrm{keV}$. For carbonnegative ion implantation, the contact angle did not show a significant change.

The ratio of introduced oxygen atoms to carbon atoms of substrate, $\mathrm{O} / \mathrm{C}$, was obtained from the ratio $\mathrm{O}_{1 s}$ and $\mathrm{C}_{1 s}$ spectra in $\mathrm{x}$-ray photoelectron spectroscopy (XPS). Figure 3 shows the $\mathrm{O} / \mathrm{C}$ ratio as a function of the ion dose for (a) $\mathrm{Ag}^{-}$ at $20 \mathrm{keV}$ and (b) $\mathrm{C}^{-}$at $5 \mathrm{keV}$. For the unimplanted NTPS, the $\mathrm{O} / \mathrm{C}$ ratio is almost zero. In the $\mathrm{Ag}^{-}$implantation of Fig. $3(\mathrm{a})$, oxygen atoms were introduced even at a low dose of $1.0 \times 10^{14} \mathrm{ions} / \mathrm{cm}^{2}$. For $\mathrm{Cu}$ implantation, a similar introduc-

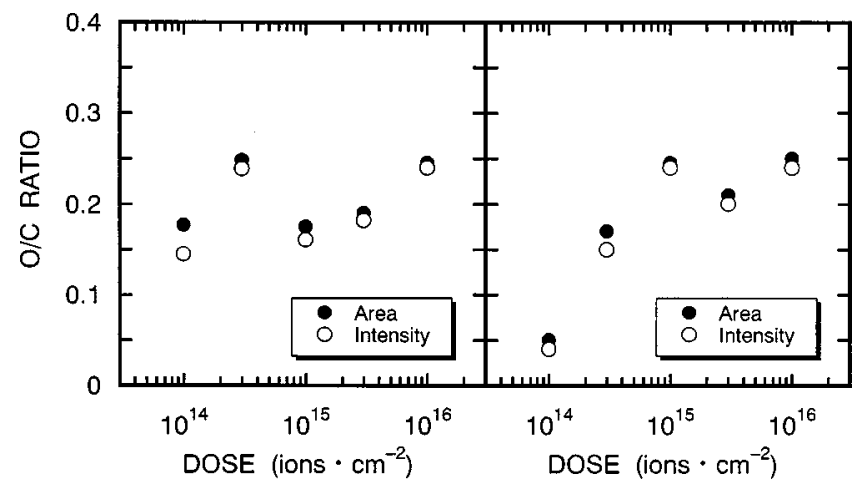

(a) $\mathrm{Ag}^{-}: 20 \mathrm{keV}$

(b) $\mathrm{C}^{-}: 5 \mathrm{keV}$

FIG. 3. The change of $\mathrm{O} / \mathrm{C}$ ratio as a function of the ion dose for (a) $\mathrm{Ag} / \mathrm{NTPS}$ at $20 \mathrm{keV}$ and (b) C/NTPS at $5 \mathrm{keV}$. In the figure, solid and open circles indicate the peak height ratio and area ratio in XPS $\mathrm{O}_{1 s}$ and $\mathrm{C}_{1 s}$ spectra, respectively. 




(a) p-Ag/NTPS



(c) p-C/NTPS

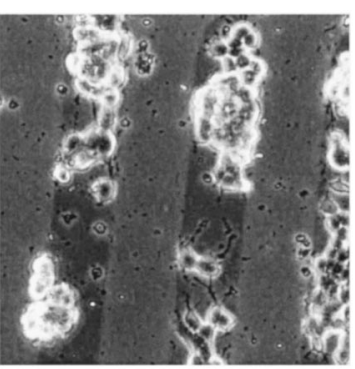

(b) p-Cu/NTPS



(d) control

(Collagen/TCPS)
FIG. 4. Phase-contrast micrographs of nerve cells of PC-12h cultured for four days (two days culture without NGF and another two days in the presence of NGF) on (a) $p$-Ag/NTPS at $20 \mathrm{keV}$, (b) $p$-Cu/NTPS at $15 \mathrm{keV}$, (c) $p$-C/NTPS at $10 \mathrm{keV}$ and (d) control (collagen TCPS, unimplanted). Dark strips in (a), (b) and (c) show the ion-implanted region with a width of $60 \mu \mathrm{m}$.

tion was observed. In the $\mathrm{C}^{-}$implantation of Fig. 3(b), O/C ratio gradually increased from $1.0 \times 10^{14}$ to 1.0 $\times 10^{16}$ ions $/ \mathrm{cm}^{2}$.

The decrease in contact angle after ion implantation into polymer is considered to be due to the formation of functional groups such as $\mathrm{C}-\mathrm{O}$ and $\mathrm{C}=\mathrm{O}$ by introducing oxygen atoms. This reason can explain the decrease of contact angle for $\mathrm{Ag}^{-}$and $\mathrm{Cu}^{-}$implantation. Although the contact angle did not change for $\mathrm{C}^{-}$implantation, the functional groups were formed.

\section{B. Nerve cell attachment and neurite extension}

Figure 4 shows phase-contrast optical micrographs of PC-12h cells cultured for four days on pattern negative ion implanted NTPS samples of (a) $p$-Ag/NTPS at $20 \mathrm{keV}$, (b) $p$-Cu/NTPS at $15 \mathrm{keV}$, (c) $p$-C/NTPS at $10 \mathrm{keV}$, and a control of (d) collagen/TCPS. In the images of Figs. 4(a), 4(b) and 4(c), the dark stripped area is an ion-implanted region on NTPS. It is clear that the nerve cells of PC-12h were selectively attached only on the ion-implanted region for all ion species, and that neurites outgrowth and extension are observed only within the same region. In comparison with the image of Fig. 4(d), PC-12h cells can normally differentiate to outgrow and extend neurites on the ion-implanted surface of polystyrene as well as on collagen of the control sample. For detailed neurite extension, the long neurites extension is observed on $p$-Ag/NTPS and $p$-C/NTPS rather than that on $p$ - $\mathrm{Cu} / \mathrm{NTPS}$.

Among the samples with respect to ion species, there was no significant difference among samples with different ion species for the cell attachment. This result of nerve-cell attachment on $p$-Ag/NTPS well agreed to that for human umbilical vascular cells (HUVEC) in our previous research. ${ }^{8}$ For $\mathrm{C}^{-}$implanted sample, cell attachment and neurite extension were also observed although the contact angle did not change. This suggests that we do not expect such biocompatibility only from the data of the contact angle. It is considered to be more important information whether functional groups can be formed or exist on the surface. They are required for absorption of some kinds of proteins, those that form the footing site for cell attachment and neurite extension. For the difference on neurite length, the reason is not clear yet. It may be considered to be due to the density of formed functional groups.

The results mean any ions can be used in ion implantation for improving biocompatibility. In order to introduce dense functional groups on the surface for efficient modification, the heavier ion is useful. Carbon atom is the main element of polystyrene, and carbon ion can efficiently transfer its kinetic energy to atoms on the surface and to make many defects. Therefore, information on what kinds of elements compose the substrate except hydrogen would be useful. In order to investigate the doping effect of a heavy metal element, ions of the element can be used for surface modification.

\section{CONCLUSIONS}

Pattern ion implantation into a polystyrene surface was found to be able to control the attachment position of nerve cells and also to draw a shape line between the neuriteextension region and the prohibited region by introducing functional groups such as $\mathrm{C}-\mathrm{O}$ and $\mathrm{C}=\mathrm{O}$ due to a radiation effect. For ion species, any ion would be used except the hydrogen ion. In conclusion, pattern ion implantation is considered as a promising method to form an artificially designed neural network for studying information processing in biological neural systems, in the neuroscience field and about the influence of heavy metals to neuron in the medical field.

${ }^{1}$ S. B. Carter, Nature (London) 213, 256 (1967)

${ }^{2}$ K. Torimitsu and A. Kawana, Dev. Brain Res. 51, 128 (1990).

${ }^{3}$ A. Kawana, Oyo Butori (in Japanese) 61, 1031 (1992).

${ }^{4}$ Y. Suzuki, M. Kusakabe, K. Kusakabe, H. Akiba, and M. Iwaki, Nucl. Instrum. Methods Phys. Res. B 59/60, 698 (1991).

${ }^{5}$ Y. Suzuki, M. Kusakabe, J. S. Lee, M. Kusakabe, M. Iwaki, and H. Sasabe, Nucl. Instrum. Methods Phys. Res. B 65, 142 (1992).

${ }^{6}$ H. Tsuji, H. Satoh, S. Ikeda, Y. Gotoh, and J. Ishikawa, Nucl. Instrum. Methods Phys. Res. B 141, 197 (1998).

${ }^{7}$ H. Tsuji, H. Satoh, S. Ikeda, N. Ikemoto, Y. Gotoh, and J. Ishikawa, Surf. Coat. Technol. 103/104, 124 (1998).

${ }^{8}$ H. Tsuji, H. Satoh, S. Ikeda, S. Ikemura, Y. Gotoh, and J. Ishikawa, Nucl. Instrum. Methods Phys. Res. B 128, 1136 (1999).

${ }^{9}$ H. Sato, H. Tsuji, S. Ikeda, N. Ikemoto, J. Ishikawa, and S. Nishimoto, J. Biomed. Mater. Res. 44, 22 (1999).

${ }^{10}$ H. Tsuji, Y. Toyota, J. Ishikawa, S. Sakai, Y. Okayama, and S. Nagumo, Ion Implantation Technology-94 (Elsevier, New York, 1995), p. 612.

${ }^{11}$ H. Tsuji, J. Ishikawa, S. Ikeda, and Y. Gotoh, Nucl. Instrum. Methods Phys. Res. B 127/128, 278 (1997).

${ }^{12}$ J. Ishikawa and H. Tsuji, Nucl. Instrum. Methods Phys. Res. B 74, 118 (1993).

${ }^{13} \mathrm{H}$. Tsuji, T. Taya, J. Ishikawa, and T. Takagi, Proceedings of the International Ion Engineering Congress, ISIAT'83\&IPAT'83, edited by T. Takagi (IEE of Japan, 1983), Vol. 1, p. 141. 